\title{
Bacillus cereus Typhlitis in a Patient with Acute Myelogenous Leukemia: A Case Report and Review of the Literature
}

\author{
James D. Denham $\mathbb{D},{ }^{1}$ Sowmya Nanjappa $\mathbb{D}^{1,2}$ and John N. Greene $\mathbb{D}^{1,2}$ \\ ${ }^{1}$ Morsani College of Medicine, University of South Florida, Tampa, FL, USA \\ ${ }^{2}$ H. Lee Moffitt Cancer Center, Tampa, FL, USA \\ Correspondence should be addressed to James D. Denham; jdenham@health.usf.edu
}

Received 13 December 2017; Accepted 18 February 2018; Published 11 March 2018

Academic Editor: Gernot Walder

Copyright (c) 2018 James D. Denham et al. This is an open access article distributed under the Creative Commons Attribution License, which permits unrestricted use, distribution, and reproduction in any medium, provided the original work is properly cited.

\begin{abstract}
Bacillus cereus is a Gram-positive rod that is now recognized as a rare cause of frank disease in the neutropenic hematologic malignancy patient. Because this pathogen is rarely isolated in clinical specimens, no large studies exist to guide the management of these acutely ill patients. Individual case reports and case series exist in the literature describing various clinical manifestations of $B$. cereus in the neutropenic patient including bacteremia/septicemia, pneumonia, meningitis/encephalitis, hepatic abscesses, and gastritis. In this report, we describe a case of typhlitis caused by B. cereus in a 74-year-old female with recently diagnosed acute myelogenous leukemia (AML), and we summarize the available English language literature to draw tentative conclusions regarding the clinical manifestations of this organism.
\end{abstract}

\section{Background}

Bacillus cereus is a Gram-positive, spore-forming rod that classically causes food poisoning via plasmid-encoded toxins. Life-threatening infections with $B$. cereus have been reported in patients with hematologic malignancies, primarily as these patients experience neutropenia following the initiation of induction chemotherapy. Here, we report a case of B. cereus colitis/typhlitis in a neutropenic patient with acute myelogenous leukemia (AML), and we summarize the literature regarding the infectious manifestations of $B$. cereus in patients with hematologic malignancies. This is the second reported case of $B$. cereus typhlitis.

\section{Case Report}

A 74-year-old Caucasian female with a 2-month history of pancytopenia and a preliminary diagnosis of myelodysplastic syndrome (MDS) presented to our tertiary cancer treatment facility for further evaluation. A repeat bone marrow biopsy was performed and demonstrated $26 \%$ blasts, and the patient was formally diagnosed with acute myelogenous leukemia
(AML). One month later, the patient was admitted to our facility for induction " $7+3$ " chemotherapy with sorafenib. On admission, the patient was found to have mild pancytopenia with an absolute neutrophil count (ANC) between 500 and 1,000 cells $/ \mu \mathrm{L}$ but had no specific complaints and an unremarkable physical examination.

Following initiation of chemotherapy, antimicrobial prophylaxis with ciprofloxacin, acyclovir, and micafungin was begun, and nebulized amphotericin B was added because computed tomography (CT) scan of the chest demonstrated several ground-glass infiltrates with no pulmonary symptoms. The ground-glass infiltrates remained stable on follow-up CT scan. Mild hemoptysis developed and empiric cefepime was begun in place of oral ciprofloxacin on the 8th day of neutropenia due to fever. She remained stable and well appearing until the 10th day of neutropenia when nonbloody diarrhea without abdominal pain developed. Clostridium difficile stool polymerase chain reaction (PCR) analysis was negative. Loperamide was administered to help reduce the diarrhea.

Diarrhea persisted despite escalating doses of loperamide, and on the 13th day of neutropenia, right upper 
TABle 1: Summary of literature review findings.

Reference Number

\author{
Uchino et al.
}

Frankard et al.

Marley et al.

Le Scanff et al.

Inoue et al.

Ginsburg et al.

Musa et al.

Akiyama et al.
Summary

This article reports 13 cases of septicemia caused by B. cereus. Uchino et al. report a case fatality rate of $25 \%$ and found that the presence of liver and CNS involvement portended a poorer prognosis. Clindamycin resistance was found to be high in this series (76.9\%), and Uchino et al. recommend against using clindamycin and carbapenems in the empiric treatment of $B$. cereus infections.

This article reports a case of pneumonia caused by $B$. cereus in the context of a neutropenic patient with ALL. This patient was treated with VCN and demonstrated clinical improvement. She was readmitted 3 weeks later with a recurrent pneumonia due to Streptococcus spp., and she expired due to this later infection.

Marley et al. report a case of meningoencephalitis occurring in a patient undergoing induction chemotherapy for AML. The patient became febrile with an ANC of $20 / \mathrm{mm}^{3}$. Over several hours, the patient experienced a rapid deterioration of mentation, and a CT scan revealed multiple enhancing lesions in the brain. The patient died 12 hours following the onset of neurological symptoms. Autopsy additionally found multiple liver abscesses.

Le Scanff et al. report a case of necrotizing gastritis in a female patient with AML 63 days following induction chemotherapy initiation. The patient developed severe epigastric pain and massive hematemesis leading to hemodynamic instability; blood and gastric mucosal cultures revealed B. cereus. This patient was successfully treated with combination of VCN and imipenem.

This substantial article reports 23 cases of $B$. cereus bacteremia in HM patients, with 12 of these patients developing a frank sepsis. Inoue reports a case fatality rate of $25 \%$ for B. cereus sepsis. Further, independent risk-factor analysis concluded that a low ANC, CV catheterization, and CNS symptoms were all significantly associated with a poorer prognosis.

Ginsburg et al. report a fatal case of B. cereus sepsis that arose in a 22-year-old male following induction chemotherapy for AML. On hospital day 5 , the patient became febrile and neutropenic and a CT scan revealed findings consistent with colitis, but stool C. difficile testing remained negative. His symptoms resolved by hospital day 14 with metronidazole and imipenem. However, 6 days later, he developed diffuse abdominal pain and blood cultures revealed B. cereus. VCN and ampicillin were added, but a new CT revealed pancolitis and multiple hypodense lesions in the liver.

The patient expired on the thirty-fourth hospital day. Autopsy confirmed the presence of $B$. cereus in the liver microabscesses.

This case series describes 3 patients, 2 with AML and 1 with ALL, who develop septicemia due to $B$. cereus. All 3 patients died despite treatment with amikacin. Additionally, all 3 patients developed a similar syndrome of abnormal posturing and clinical signs of brain stem death. 1 patient in this case series was found to have elevated liver enzymes and serum bilirubin perimortem.

Akiyama et al. report a case of fatal B. cereus septicemia in a 64-year-old patient with AML undergoing induction chemotherapy. Autopsy revealed a necrotizing

infection in the leptomeninges of the brain and spinal cord and numerous microabscesses of the liver. B. cereus was present within these lesions.

$\mathrm{CNS}=$ central nervous system; $\mathrm{ALL}=$ acute lymphoblastic leukemia; $\mathrm{VCN}=$ vancomycin; $\mathrm{AML}=$ acute myelogenous leukemia. $\mathrm{ANC}=$ absolute neutrophil count. $\mathrm{CT}=$ computed tomography. $\mathrm{HM}=$ hematologic malignancy. $\mathrm{CV}=$ central venous. $\mathrm{C}$. difficile = Clostridium difficile.

quadrant abdominal pain developed and metronidazole $500 \mathrm{mg}$ twice daily was initiated. CT scan of the abdomen and pelvis revealed cecal wall thickening associated with fat stranding and right paracolic gutter fluid collectionfindings consistent with typhlitis. Labs revealed an ANC of less than 500 cells $/ \mu \mathrm{L}$. Stool cultures obtained the following day revealed a preponderance of Bacillus cereus with the absence of usual enteric flora. Physical examination then demonstrated dull periumbilical pain upon palpation and a sharper pain in the right lower quadrant of the abdomen. Metronidazole was changed to clindamycin $450 \mathrm{mg}$ thrice daily. During the next several days, the abdominal pain and diarrhea resolved. The patient's later hospital course was complicated by vancomycin-resistant Enterococcus (VRE) bacteremia that developed after 21 days of neutropenia and was treated with central venous catheter removal and linezolid $600 \mathrm{mg}$ twice per day. She would finish the 2-week linezolid course outpatient; she was discharged afebrile after 30 hospital days with an ANC of 3.28 cells/ $\mu$ L. Complete clinical remission of the AML was ultimately achieved following 2 rounds of consolidation chemotherapy.

\section{Discussion}

Typhlitis, also known as neutropenic enterocolitis (or, rarely, ileocecal syndrome) is the most common cause of fever and 
TABLE 2: Conclusions for Bacillus cereus infection in neutropenic hematologic malignancy patients.

\begin{tabular}{|c|c|c|}
\hline & Conclusion & Reference Number \\
\hline 1 & $\begin{array}{l}\text { Neutropenia is the primary risk factor for infection, as opposed to } \\
\text { intoxication, with } B \text {. cereus }\end{array}$ & {$[5-12]$} \\
\hline 2 & $\begin{array}{l}\text { B. cereus has a predilection for the CNS and the liver; involvement } \\
\text { of either of these two organ systems portends a poor prognosis }\end{array}$ & {$[5,7,9-12]$} \\
\hline 3 & The case fatality rate for B. cereus sepsis appears to be $25 \%$ & {$[5,9]$} \\
\hline 4 & $\begin{array}{l}\text { The most commonly used definitive therapy for } B \text {. cereus infection } \\
\text { appears to be vancomycin with or without carbapenem }\end{array}$ & {$[5-10]$} \\
\hline
\end{tabular}

CNS $=$ central nervous system.

abdominal tenderness in the neutropenic patient [1]. Historically, Clostridium septicum was pointed to as a common etiologic agent of typhlitis [2]. It is known, however, that the microbiologic etiology of this disease is broad and diverse, with an 18-year study in leukemic children finding that $84 \%$ and $16 \%$ of cases being caused by bacteria and fungi, respectively [3]. Currently, there exist no high-quality casecontrol, cohort, or randomized controlled trials to guide clinicians in the best management of typhlitis [4].

The isolation of $B$. cereus from clinical specimens was historically considered contamination. However, it is now clear from the literature that $B$. cereus can cause a wide spectrum of disease in the neutropenic patient. Our patient, a 74-year-old female undergoing induction chemotherapy for AML, was found to have typhlitis with stool culture confirming the presence of $B$. cereus in the colon with a notable absence of the usual enteric flora. The initiation of clindamycin led to a rapid improvement in our patient's symptoms. Our patient's blood cultures remained sterile during the episode of typhlitis.

On review of the literature, we found a handful of reports describing the various clinical manifestations of $B$. cereus (Table 1). The most common manifestation was sepsis. As reported by Uchino et al., patients who develop central nervous system (CNS) symptoms or who have bacterial involvement of the liver tend to have a worse prognosis $[5,9]$. Indeed, from our review, it is clear that while $B$. cereus bacteremia can result in the seeding of almost any organ, B. cereus seems to preferentially seed the brain/meninges and the liver [5, 7, 9-12]. CNS involvement is most commonly multifocal and leads to a rapid deterioration in mental status and frequently leads to development of a comatose state. Liver involvement is often asymptomatic but can manifest on CT scan as multiple subcentimeter, hypodense lesions. Pathologic analysis of the liver at autopsy reveals microabscesses with large Gram-positive rods present within the lesions $[10,12]$.

The management of the neutropenic patient with cultures positive for $B$. cereus presents a clinical dilemma as no large studies exist to guide the management of these critically ill patients. From our review of the available English language literature, some tentative conclusions may be drawn regarding this organism in the context of the neutropenic hematologic malignancy patient. These conclusions are summarized in Table 2.

\section{Conflicts of Interest}

The authors declare that there are no conflicts of interest regarding the publication of this paper.

\section{Authors' Contributions}

All authors have participated significantly in writing of this manuscript and approve of its content. The final manuscript has been seen and approved by all authors.

\section{References}

[1] B. D. Badgwell, J. N. Cormier, C. J. Wray et al., "Challenges in surgical management of abdominal pain in the neutropenic cancer patient," Annals of Surgery, vol. 248, no. 1, pp. 104-109, 2008.

[2] G. D. Rifkin, "Neutropenic enterocolitis and Clostridium septicum infection in patients with agranulocytosis," Archives of Internal Medicine, vol. 140, no. 6, pp. 834-835, 1980.

[3] J. A. Katz, M. L. Wagner, M. V. Gresik, D. H. Mahoney, and D. J. Fernbach, "Typhlitis: an 18-year experience and postmortem review," Cancer, vol. 65, no. 4, pp. 1041-1047, 1990.

[4] M. Gorschlüter, U. Mey, J. Strehl et al., "Neutropenic enterocolitis in adults: systematic analysis of evidence quality," European Journal of Haematology, vol. 75, no. 1, pp. 1-13, 2005.

[5] Y. Uchino, N. Iriyama, K. Matsumoto et al., "A case series of Bacillus cereus septicemia in patients with hematological disease," Internal Medicine, vol. 51, no. 19, pp. 2733-2738, 2012.

[6] J. Frankard, R. Li, F. Taccone et al., "Bacillus cereus pneumonia in a patient with acute lymphoblastic leukemia," European Journal of Clinical Microbiology \& Infectious Diseases, vol. 23, no. 9, pp. 725-728, 2004.

[7] E. F. Marley, N. K. Saini, C. Venkatraman, and J. M. Orenstein, "Fatal Bacillus cereus meningoencephalitis in an adult with acute myelogenous leukemia," Southern Medical Journal, vol. 88, no. 9, pp. 969-972, 1995.

[8] J. Le Scanff, I. Mohammedi, A. Thiebaut, O. Martin, L. Argaud, and D. Robert, "Necrotizing gastritis due to Bacillus cereus in an immunocompromised patient," Infection, vol. 34, no. 2, pp. 98-99, 2006.

[9] D. Inoue, Y. Nagai, M. Mori et al., "Fulminant sepsis caused by Bacillus cereus in patients with hematologic malignancies: analysis of its prognosis and risk factors," Leukemia \& Lymphoma, vol. 51, no. 5, pp. 860-869, 2010.

[10] A. S. Ginsburg, L. G. Salazar, L. D. True, and M. L. Disis, "Fatal Bacillus cereus sepsis following resolving neutropenic 
enterocolitis during the treatment of acute leukemia," American Journal of Hematology, vol. 72, no. 3, pp. 204-208, 2003.

[11] M. O. Musa, M. Al Douri, S. Khan, T. Shafi, A. Al Humaidh, and A. M. Al Rasheed, "Fulminant septicaemic syndrome of Bacillus cereus: three case reports," Journal of Infection, vol. 39, no. 2, pp. 154-156, 1999.

[12] N. Akiyama, K. Mitani, Y. Tanaka et al., "Fulminant septicemic syndrome of Bacillus cereus in a leukemic patient," Internal Medicine, vol. 36, no. 3, pp. 221-226, 1997. 


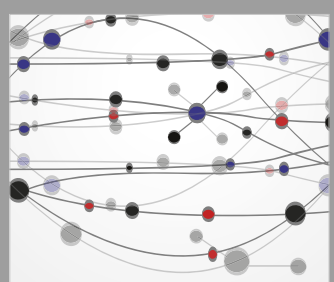

The Scientific World Journal
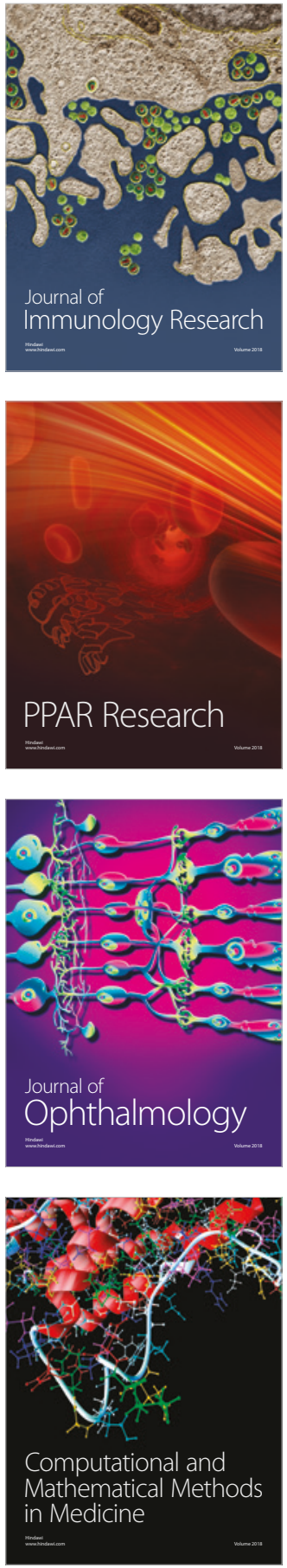

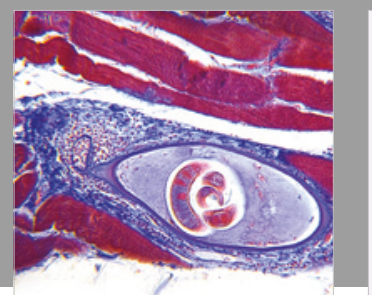

Gastroenterology Research and Practice

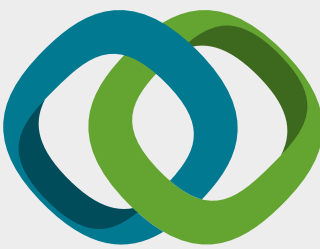

\section{Hindawi}

Submit your manuscripts at

www.hindawi.com
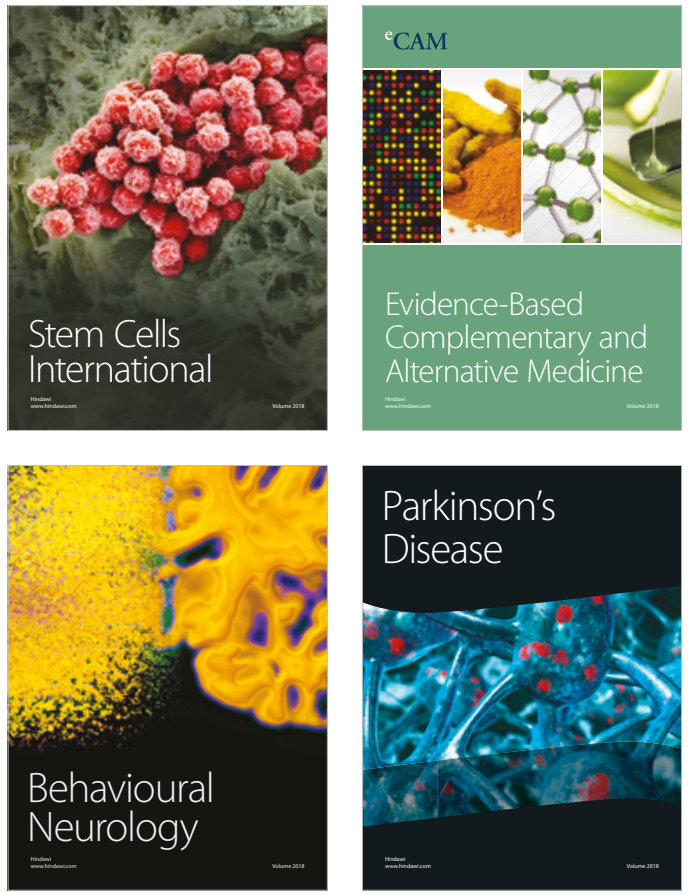

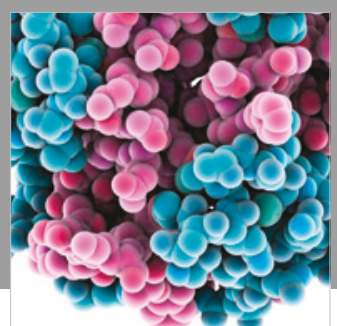

ournal of

Diabetes Research

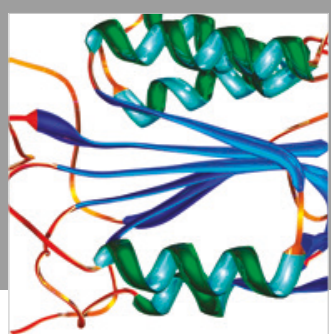

Disease Markers
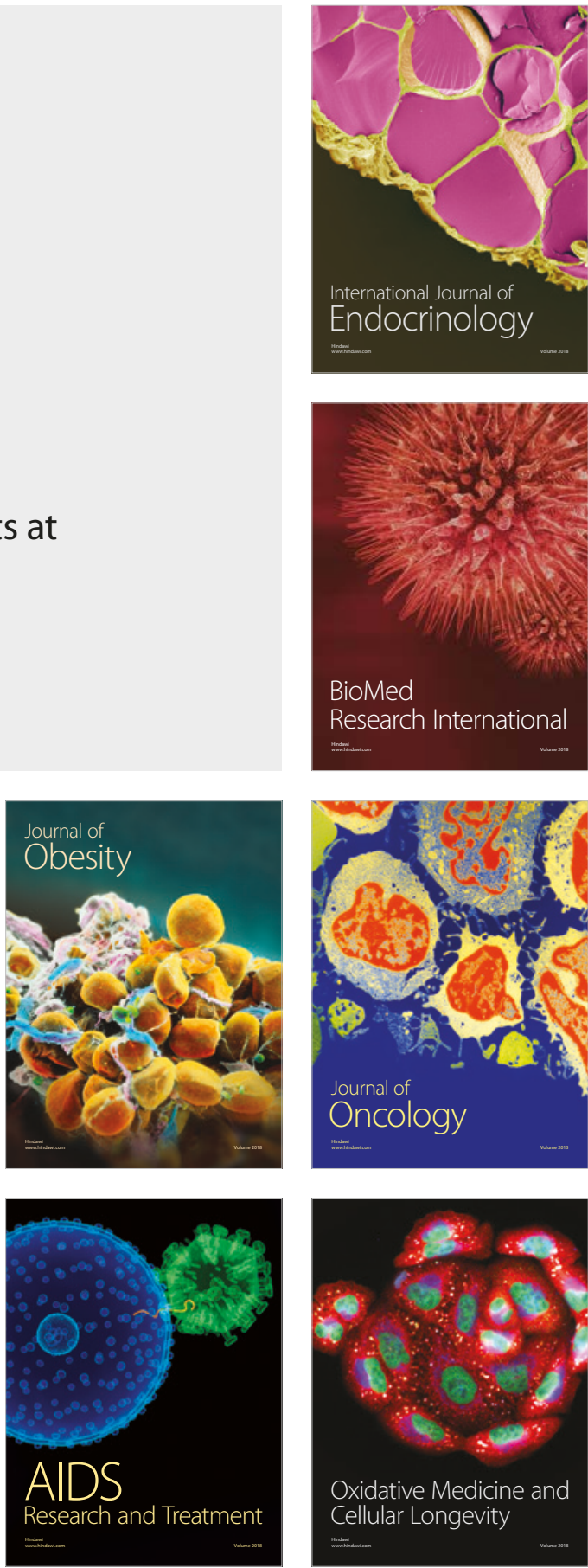